\title{
Reliability and Sex Differences in a Coordination Test of a Tracking Moving Target with the Center of Foot Pressure
}

\author{
Haruka Kawabata $^{1 *}$, Shinichi Demura ${ }^{1}$, Masanobui Uchiyama ${ }^{2}$ \\ ${ }^{1}$ Graduate School of Natural Science and Technology, Kanazawa University, Kanazawa, Japan \\ ${ }^{2}$ Akita Prefectural University, Akita, Japan \\ Email: "sjskd631@ybb.ne.jp
}

Received April 30 ${ }^{\text {th }}$, 2012; revised May $28^{\text {th }}$, 2012; accepted June $7^{\text {th }}, 2012$

\begin{abstract}
This electronic document is a "live" template. The various components of your paper (title, text, heads, etc.) are already defined on the style sheet, as illustrated by the portions given in this document.
\end{abstract}

Keywords: Center of Pressure; Coordination; Tracking Test; Reliability; Sex Differences

\section{Introduction}

\section{Coordination}

Coordination is the ability to accurately accomplish physical activity in a timely way, and is closely related to nerve function. It is generally considered to consist of elements such as elaboration, dexterity, accuracy, skill, and agility (Ferslew et al., 1982). It is necessary to coordinate the exertion of muscle strength to perform movements efficiently.

\section{Coordination Test}

Upper limb movements such as using chopsticks and writing require coordination. Hence, coordination tests such as the Purdue Pegboard test (Buddenberg \& Davis, 2000) and the Moving Beans with Tweezers test (Shigematsu et al., 2001) have been developed (Ferslew et al., 1982; Noguchi et al., 2006; Nakafuji \& Tsuji, 2001). Nagasawa et al. (2002) developed a controlled force exertion (CFE) test pursuing the changing demand values, appearing as sinusoidal and quasi-random waveforms on a personal computer, by grip strength exertion. These tests evaluate mainly upper limb coordination. However, since daily life and sports activities are generally performed standing upright, they require the coordination of the whole body. Visual, somatosensory, and sensory motor system functions contribute to efficiently perform movements. Moreover, in movements involving the whole body, many skeletal muscles are needed to maintain posture balance. However, not many tests have been developed that rationally evaluate coordination of the whole body.

Recently, it has become possible to record the center of foot pressure (COP), which is projected at the body center of gravity on the plantar surface. As a result of the central nervous system (CNS) receiving feedback from visual, vestibular, and somatic sensations, the COP during standing always sways slightly. The total length and area of body sway have been widely used an index of balance function disorder and static balance ability. According to previous studies on COP sway (Ekdahi et al., 1982; Goldie, 1989; Hattori et al., 1992; Dickstein et al., 1993; Yamaji et al., 2001), the reliability of the COP parameters during standing is high. Recently, a balance measurement devive

\footnotetext{
"Corresponding author.
}

has been developed that can follow a randomly moving target by voluntary changes in COP. It is assumed that well-performing individuals who can coordinate their entire body can also meet their COP to a moving target on the above device. In short, this device can evaluate the coordination of the whole body. Yoshida et al. (1997) developed the test that tracks a moving target by COP. However, in their test the target moves linearly (back and forth, left and right) and constantly, the subjects can predict the target's movements. A test involving an unpredictably moving target will be able to more accurately evaluate the coordination of the whole body.

\section{Reliability of the New Test and Sex Difference}

On the other hand, when developing a new test, it is necessary to establish the measurement method and to determine appropriate evaluation parameters. In addition, it is essential to examine the reliability of the parameters. The practice effect is often found in coordination tests and performances improve with every trial (Noguchi et al., 2009). Hence, the practice effect may also be found in a coordination test involving the whole body tracking a moving target by the COP. In additional, Nagasawa et al. (2002) clarified that males perform better than females in the controlled force exertion (CFE) test for both hands. Hence, they (Nagasawa et al., 2009: Nagasawa et al., 2010) determined the standards of the CFE test according to sex. The existence of standards is one important condition of a good test. If sexual differences are found in the test, it is necessary to make up a different standard according to each sex. Hence, it also is important to examine whether a sex difference is found in advance.

During movements using whole body, it is required to cooperatively exert many skeletal muscles of the whole body to keep posture balance. However, tests that rationally evaluate coordination of the whole body have little been developed. Recently, a balance measurement device, which can pursue voluntary the randomly moving targets by the COP, has developed. It is assumed that superior persons in coordination of whole body can meet right the COP to a moving target on the above device. In short, it is considered to be possible to evaluate coordination of the whole body by a test using the above device.

However, a size of reliability in this test which is one important 
condition of the test is unclear. Hence, this study aimed to examine reliability and sex difference of the new coordination test pursuing the randomly moving target by the COP.

\section{Method}

\section{Subjects}

The subjects were 30 healthy people consisting of 15 males (age $23.9 \pm 4.7 \mathrm{yrs}$, height $171.5 \pm 4.0 \mathrm{~cm}$, weight $66.9 \pm 8.1 \mathrm{~kg}$ ) and 15 females (age $20.7 \pm 3.1$ yrs, height $161.1 \pm 5.5 \mathrm{~cm}$, weight $54.6 \pm 5.3 \mathrm{~kg}$ ). No participant reported previous leg injuries or nerve disorder of lower limbs. Prior to measurement, the purposes and procedures of this study were explained in detail, and written informed consent was obtained from all subjects. The protocol of this study was approved by the Kanazawa University Department of Education Ethical Review Board.

\section{Test of Whole Body Coordination by Tracking a Moving Target with COP}

In the motor task of pursuing the randomly moving target with the COP, various nervous controls were needed by the subjects. First, after the motor area and the cerebellum determined the tactics that make the COP track a moving visual target, they transfer the commands coordinating the contracture of muscles of each part of the body through spinal cord and alpha motor neurons, and determine speed, duration, and accuracy of the motion (Hanatsch \& Langer, 1985). Body balance is maintained by the involuntary postural control derived from the brain stem, in addition to the above stated voluntary control. In short, since tracking by the COP is performed coordinately by voluntary movements of the whole body and involuntary movements that maintain balance, it was assumed that whole body coordination can be evaluated by the tracking task. Hence, it is judged that subjects with a smaller tracking error between their COP and the moving target are able to better match their COP to a moving target. In this study, subjects with a smaller totalerror, within a certain time, were assumed to have superior coordination of the whole body.

\section{Experiment Equipment}

The measuring device used in this study was G-620 (Anima Corp., Japan). It consists of the force platform containing three sensors for the vertical load, an amplifier, and feedback display (size: 15.6 inch; resolutions: $1366 \times 768$ ). By using this device, subjects receive visual feedback information regarding their COP position through the device's monitor. Thus, they can track the randomly moving visual target on the monitor and compare it to their COP. In addition, since it can record the subject's COP position during standing on the force platform over time, the errors between the moving target and the subject's COP can be calculated.

\section{Experimental Condition}

In this study, COP position was recorded for one minute with sampling frequency $20 \mathrm{~Hz}$ (Demura et al., 2008) in each trial. The monitor for the visual feedback was placed at $1.5 \mathrm{~m}$. in front of the subject and at eye level. The moving target randomly moved within a range of $10 \mathrm{~cm}$. from the coordinate origin on an $x-y$ plane of the visual feedback monitor. The target positions in the $\mathrm{y}$ and $\mathrm{x}$ directions were programmed to fluctuate along with the substantially sinusoidal waveform (period: $12 \pm 5$ sec; amplitude: $5 \pm 5 \mathrm{~cm}$ ).

\section{Test and Test Procedure}

The subjects stood on the platform bare-footed, with their arms held comfortably at the side of the body and their eyes open (Figure 1). The randomly (by period and amplitude) moving target and the subject's COP position were shown on the feedback monitor in front of them. Then, they were instructed to track the moving target with their COP (Figure 2). After one practice trial, subjects carried out five trials with a break of one minute between each trial. To exclude the influence of measurement errors immediately after the first and the final phases, COP data obtained from the first $20 \mathrm{sec}$. and the last $10 \mathrm{sec}$. were omitted, in accordance with previous studies on CFE (Nagasawa et al., 2002; Riviere \& Thakor, 1996). In short, data from 30 seconds were used as an evaluation parameter.

\section{Statistical Analysis}

A two-way analysis of variance (ANOVA) was used to test mean differences of trial and sex factors. Mean differences among trials was tested by one-way analysis of variance. Intra class correlation coefficient was calculated to examine reliability of the test. The level of significance was set a priori to .05 .

\section{Results}

Table 1 shows the means and standard deviations of five trials according to sex. A result of two-way ANOVA showed insignificance in main effect of trial and sex factors. The whole mean of 5 trials was $1444.0 \pm 277.6$ in males and $1481.6 \pm$ 313.2 in females, and significant sex difference was not found.

Figure 3 shows the means, standard deviations, one-way ANOVA, and ICC of all five trials and of the first three trials based on data pooled of males and females. The mean of five trials showed insignificant difference, and the ICC of the above trials was 0.68 and 0.75 , being somewhat higher in the latter. Table 2 shows the means of three trials after excluding maxi-
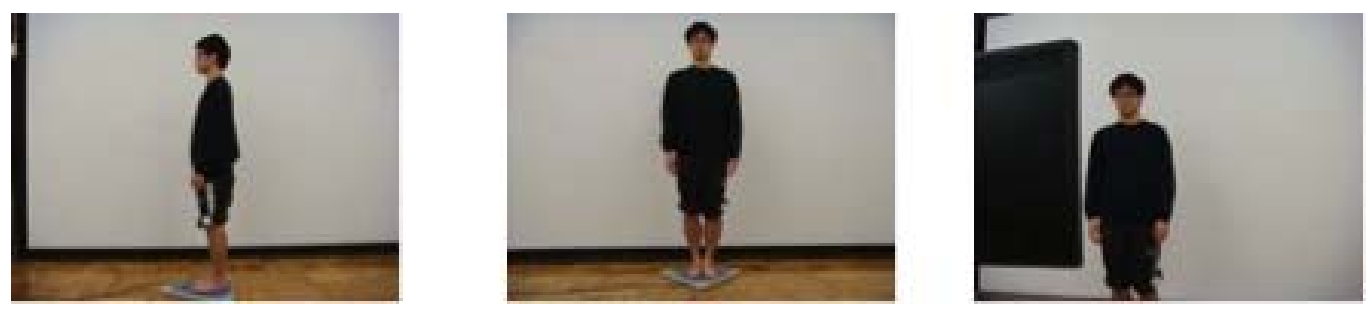

Figure 1.

Posture of the subject during the test. 


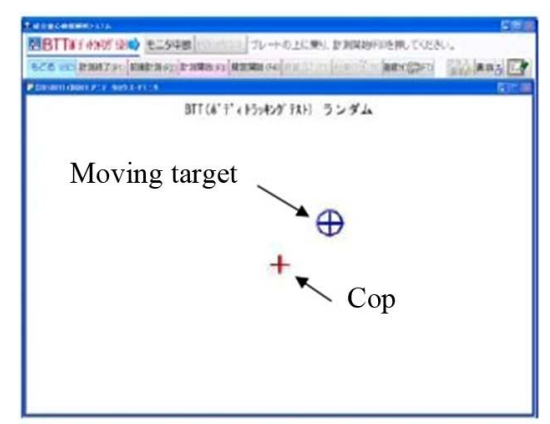

$(\mathrm{cm})$

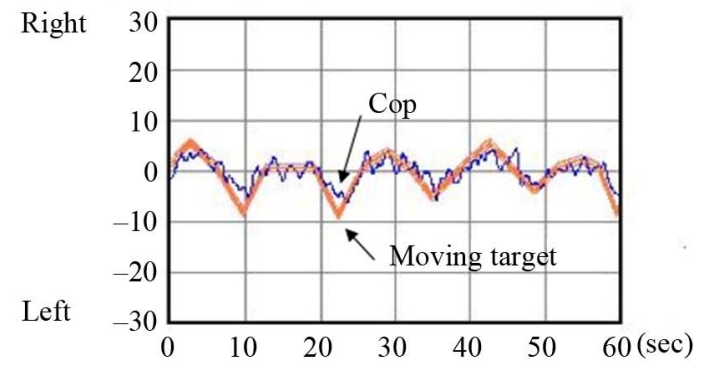

Figure 2.

Screen during the test and test results.

Table 1.

The errors between moving target and COP.

\begin{tabular}{|c|c|c|c|c|c|c|c|c|c|c|c|c|c|c|c|c|c|}
\hline & \multicolumn{2}{|c|}{ Trial 1} & \multicolumn{2}{|c|}{ Trial 2} & \multicolumn{2}{|c|}{ Trial 3} & \multicolumn{2}{|c|}{ Trial 4} & \multicolumn{2}{|c|}{ Trial 5} & \multicolumn{4}{|c|}{ 1-5 Trials } & \multicolumn{3}{|c|}{ ANOVA } \\
\hline & Mean & SD & Mean & SD & Mean & SD & Mean & SD & Mean & SD & Mean & SD & Max & Min & $\mathrm{F}$ & $p$ & ICC \\
\hline Male (n = 15) & 1487.2 & 265.4 & 1413.3 & 267.5 & 1476.3 & 328.5 & 1396.0 & 276.2 & 1447.4 & 250.4 & 1444.0 & 277.6 & 1723.0 & 1239.5 & 0.68 & 0.61 & 0.66 \\
\hline Female $(n=15)$ & 1518.0 & 331.1 & 1493.5 & 299.7 & 1472.4 & 271.8 & 1457.3 & 281.9 & 1467.0 & 381.4 & 1481.6 & 313.2 & 1952.5 & 1313.8 & 0.30 & 0.88 & 0.71 \\
\hline
\end{tabular}

Note: ${ }^{*} p<.05$, Mean (cm), SD: Standard Deviation, ICC: Intraclass correlation coefficient.

mum and minimum values among the five trials. An insignificant mean difference among trials was found and the ICC was 0.86 , being somewhat higher than the above two ICCs.

\section{Discussion}

\section{Sex Difference}

This study examined sex difference and reliability of a novel whole-body coordination test where the COP is used to follow a randomly moving target. Nagasawa et al. (2000) examined a sex difference in controlled force exertion (CFE) of middle-aged and elderly subjects, and reported that the males performed better than the females. Kawabata et al. (2011) reported that in the pursuit rotor test, young men performed significantly better than young women. York \& Biederman (1990) reported that males performed better in tests that require dexterity of the hands and fingers. On the other hand, Haward and Griffin (2002) reported that a significant sex difference was found in the maximum grip strength test, but not in the Purdue Pegboard test.

In the CFE test, subjects pursue the target presented on the computer screen by grip strength exertion. In a pursuit rotor test, they track the rotating target with a metalrod (Ferslew et al., 1982; Nakafuji \& Tsuji, 2001). These tests evaluate the accuracy of grip and manipulation of upper limb movements that are frequently used in daily life, and they generally show functional lateral dominancy (Chi \& Dooling, 1977; Touwen, 1972; Noguchi et al., 2009; Kawabata et al., 2011). In the cases of the above tests, exercise experience in the past and use frequency in the present life environment may largely affect measurements.

A sex difference was not found in the tracking test by the COP used in this study. If strength and agility (speed) relate largely to the test motion, a sex difference could have been found since males are generally superior in these abilities. The novel test presented here is performed in a standing position, and since the task involved both legs, burden imposes on both legs almost evenly. From the test content, a large exertion of strength and agility is not required. Hence, it is inferred that a sex difference was not found.

\section{Reliability between Trials}

Since a sex difference was not found, reliability was examined by using pooled data of males and females. A practice effect is generally found in coordination tests (Noguchi et al., 2009), but an insignificant difference was found among means of five trials. A possible explanation is that the present coordination test is performed in static standing posture and does not require special techniques. In addition, stable data for $30 \mathrm{sec}-$ onds excluding data of the first 20 seconds and the last 10 seconds (see Methods) was used for statistical analysis. For a standard of reliability, ICC over 0.60 by Landis and Koch, (1997) and .70 by Portney \& Watkins (1983) have been reported and widely accepted. Hence, reliability of the present test is considered to be guaranteed in both the five trials (.68) and first three trials (.75). However, the latter ICC was somewhat higher. When performing the task of pursuing the moving target with COP for one minute, subjects need considerable tension, responsiveness and concentration. In addition, it takes about 10 minutes to perform the five trials, including the instructions and one practice trial. Hence, even if subjects had enough rest between trials, it is inferred that the stability of measurements in the latter trials decreased due to concentration loss and fatigue. From the present results, when considering practical application, it may be appropriate to administer three trials and use that mean as are presentative of value.

On the other hand, in the whole body reaction time test, there are cases when subjects respond predictively before the signal, or their reaction is delayed abnormally due to missing the signal or the response timing. In short, it is possible that abnormal values are measured accidentally. When using these abnormal values as measured values, means and standard deviations are greatly affected. Considering this problem, a mean excluding the maximum and minimum values after performing five or seven trials is generally used during the whole body reaction time test. Nagasawa and Demura (2004) reported that the mean 


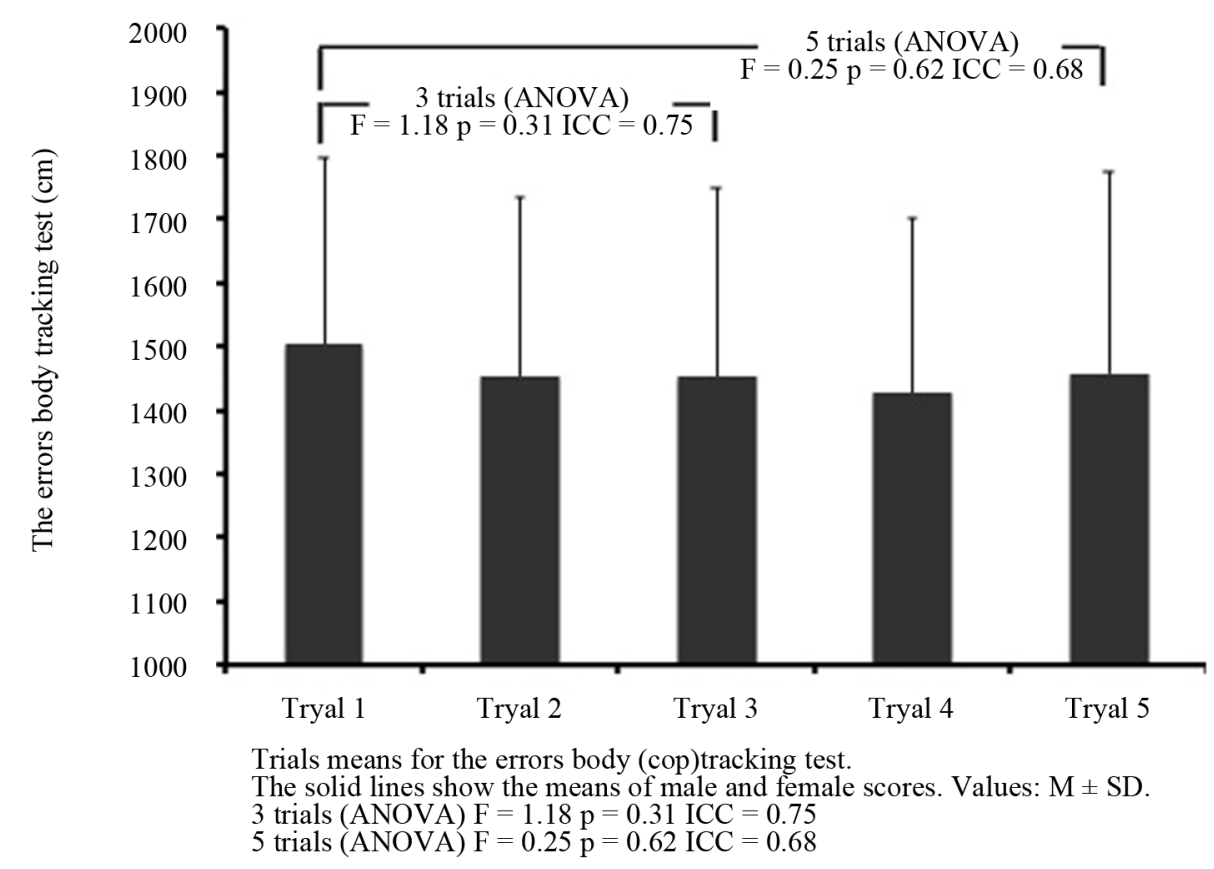

Figure 3.

Posture of the subject during the test.

Table 2.

The means of 3 trials excluding maximum and minimum values 5 trials.

\begin{tabular}{|c|c|c|c|c|c|c|c|c|c|c|c|}
\hline \multicolumn{2}{|c|}{1} & \multicolumn{2}{|c|}{2} & \multicolumn{2}{|c|}{3} & \multicolumn{2}{|c|}{3 Trials } & \multicolumn{3}{|c|}{ ANOVA } & \\
\hline Mean & SD & Mean & SD & Mean & SD & Mean & SD & $\mathrm{F}$ & $p$ & ICC & \\
\hline 1479.4 & 291.7 & 1444.0 & 282.6 & 1419.7 & 252.6 & 1447.7 & 275.6 & 2.77 & 0.07 & 0.86 & 0.07 \\
\hline
\end{tabular}

after excluding maximum and minimum values among seven trials was high in the pursuit rotor test. The ICC (.86) of three trials calculated in this study based on the above exclusion practice was higher than the other ICCs. This method may also be effective in ensuring high reliability. Previous studies on the pursuing test using the COP (Yoshida et al., 1997; Yamamoto et al., 1997; Hamann et al., 1990) evaluate the ability to follow a linearly and constantly moving target along longitudinal and lateral directions. In that test, subjects can predict target movements and adjust easily. In order to adequately evaluate whole body coordination ability, pursuing a randomly moving target as used in this study is considered to be valid.

In conclusion, this test of whole body coordination shows insignificant sex difference and has high reliability. From a perspective of practicality, it may be useful to use the mean of the three trials as an evaluation parameter after one practice trial. When prioritizing high reliability, it will be appropriate to use data excluding the maximum and minimum values.

\section{REFERENCES}

Buddenberg, L. A., \& Davis, C. (2000). Test-retest reliability of the Purdue Pegboard test. American Journal of Occupational Therapy, 54, 555-558. doi:10.5014/ajot.54.5.555

Chi, J. G., Dooling, E. C., \& Gilles, F. H. (1977). Left-right asymmetry of the temporal speech areas of human fetus. Archives of Neurology, 34, 346-348. doi:10.1001/archneur.1977.00500180040008

Dickstein, R., \& Dvir, Z. (1993). Quantitative evaluation of stance balance performance in the clinic using a novel measurement device.
Physiotherapy Canada, 45, 102-108.

Demura, S., Kitabayashi, T., \& Aoki, H. (2008). Body-sway characteristics during a static upright posture in the elderly. Journal of Physiological Anthropology and Applied Human Science, 8, 188-197.

Ekdahi, C., Jarnlo, G. B., \& Andersson, S. I. (1989). Standing barance in healthy subjects. Scandinavian Journal of Rehabilitation Medicine, 21, 187-195.

Ferslew, K. H., Manno, J. E., Manno, B. R., Vekovius, W. A., Hubbard, J. M., \& Bairnsfather, L. E. (1982). Pursuit meter II: A computer-based device for testing pursuit-tracking performance. Perceptual \& Motor Skills, 54, 779-784. doi:10.2466/pms.1982.54.3.779

Goldie, P. A., Bach. T. M., \& Evans, O. M. (1989). Force platform measures for evaluating postural control: Reliability and validity. Archives of Physical Medicine and Rehabilitation, 70, 510-517.

Kawabata, H., Demura, S., Kitabayashi, T., \& Sato, S. (2011). Gender and laterality of various coordination test. In press.

Nagasawa, Y., Demura, S., Yamaji, S., Kobayashi, H., \& Matsuzawa, J. (2000). Ability to coordinate exertion of force by the dominant hand: comparisons among university students and 65- to 78-year-old men and women. Perceptual \& Motor Skills, 90, 995-1007. doi:10.2466/pms.2000.90.3.995

Nagasawa, Y., \& Demura, S. (2002). Development of an apparatus to estimate coordinated exertion of force. Perceptual \& Motor Skills, 94, 899-913.

Nagasawa, Y., \& Demura, S. (2004) Relationships among coordinated exertion of force and performance on pegboard and pursuit rotor tests using upper limbs and fingers. Perceptual \& Motor Skills, 99, 10531060.

Nagasawa, Y., Demura, S., \& Hamasaki, H. (2009) Provisional norms by age group for Japanese males on the controlled force exertion test using a quasi-random display. Sport Sciences for Health, 5, 121-127. doi:10.1007/s11332-009-0087-5 
Nagasawa, Y., \& Demura, S. (2010) Provisional norms by age group for Japanese women on the controlled force exertion test using a quasi-random display. Perceptual \& Motor Skills, 110, 613-624. doi:10.2466/pms.110.2.613-624

Nakafuji, A., \& Tsuji, K. (2001). Learning and transfer in two perceptual-motor skills in duchenne muscular dystrophy. Perceptual \& Motor Skills, 93, 339-352.

Noguchi, T., Demura, S., Nagasawa, Y., \& Uchiyama, M. (2006). Practice effect and its difference of the pursuit rotor test by the dominant and non-dominant hands. Journal of Physiological Anthropology and Applied Human Science, 102, 265-274.

Noguchi, T., Demura, S., Nagasawa, Y., \& Uchiyama, M. (2009) Influence of measurement order by dominant and nondominant hands on performance of a pursuit-rotor task. Perceptual \& Motor Skills, 108, 905-914. doi:10.2466/pms.108.3.905-914

Hamann, R. G., Mekjavic, I., Mallinson, A. I., \& Longridge, N. S. (1992). Training effects during repeated therapy sessions of balance training using visual feedback. Archives of Physical Medicine and Rehabilitation, 73, 738-744.

Haward, B. M., \& Griffin, M. J. (2002). Repeatability of grip strength and dexterity tests and the effects of age and gender. International Archives of Occupational and Environmental Health, 75, 111-119.

Hattori, K., Staekes, J., \& Takahashi, T. (1992). The influence of age on variability of postural sway during the daytime. Japanese Journal of Human Posture, 11, 137-146.

Henatsch, H. D., \& Langer, H. H. (1985). Basic neurophysiology of motor skills in sport: A review. International Journal of Sports Medicine, 6, 2-14. doi:10.1055/s-2008-1025805

Landis, J. R., \& Koch, G. G. (1977). The measurement of observer agreement for categorical data. Biometrics, 33, 159-174. doi:10.2307/2529310

Portney, L. G., \& Watkins, M. P. (1983). Foundations of clinical research application to practice. Norwalk CT: Appleton \& Lange, 505-516.

Riviere, C. N., \& Thakor, N. V. (1996). Effects of age and disability on tracking tasks with a computer mouse: Accuracy and linearity. Journal of Rehabilitation Research and Development, 33, 6-15.

Shigematsu, R., Tanaka, K., Holland, G., Nakagaichi, M., Chang, M., Takeshima, N., Noda, F., Tanaka, Y., \& Mimura, K. (2001). Validation of the functional fitness age (FFA) index in older Japanese women. Aging, 13, 385-390.

Touwen, B. C. (1972). Laterality and dominance. Developmental Medicine \& Child Neurology, 14, 747-755. doi:10.1111/j.1469-8749.1972.tb03318.x

Yamaji, S., Demura, S., Noda, M., Nagasawa, Y., Nakada, M., \& Kitabayashi, T. (2001). The day-to-day reliability evaluating the body center of presssure in static standing posture. Equilibrium Research, 60, 217-226. doi:10.3757/jser.60.217

Yamamoto, M., Yoshida, T., Oda, M., \& Takeuchi, J. (1996). Development of a test system of dynamic postural control using a gravicorder: Body tracking test system. Equilibrium Research, 55, 262269. doi:10.3757/jser.55.262

Yoshida, T., Oda, M., Osafune, H., Miyaji, M., \& Yamamoto, M. (1997). The evaluation of tracking ability by the body tracking (BTT). Equilibrium Research, 56, 34-44.

York, J. L., \& Biederman, I. (1990). Effects of age and sex on reciprocal tappingperformance. Perceptual \& Motor Skills, 71, 675-684. doi:10.2466/pms.1990.71.2.675 